\title{
New rules on driver licensing for patients with obstructive sleep apnoea: EU Directive 2014/85/EU
}

\author{
Maria R. Bonsignore ${ }^{1}$, Winfried Randerath ${ }^{2}$, Renata Riha ${ }^{3}$, Dan Smyth ${ }^{4}$, \\ Christina Gratziou ${ }^{5}$, Marta Goncalves ${ }^{6}$ and Walter T. McNicholas ${ }^{7}$
}

Affiliations: ${ }^{1}$ DiBiMIS, University of Palermo and Institute of Biomedicine and Molecular Immunology (IBIM), National Research Council (CNR), Palermo, Italy. ${ }^{2}$ Pneumologie und Allergologie Zentrum für Schlaf - und Beatmungsmedizin, Krankenhaus Bethanien, Solingen, Germany. ${ }^{3}$ Sleep and Respiratory Medicine, University of Edinburgh, Edinburgh, UK. ${ }^{4}$ European Lung Foundation Chair. ${ }^{5}$ Medical School, Athens University, Athens, Greece. ${ }^{6}$ Institute of Public Health - University of Porto (ISPUP), Porto, Portugal. ${ }^{7}$ Dept of Respiratory and Sleep Medicine, St. Vincent's University Hospital, University College Dublin, Dublin, Ireland.

Correspondence: Maria R. Bonsignore, Dipartimento Biomedico Di Medicina Interna e Specialistica (DiBiMIS), University of Palermo, Via Trabucco 180, Palermo, Italy. E-mail: marisađibim.cnr.it

@ERSpublications

Joint ERS/ESRS working group on the EU directive on issuing driving licences in obstructive sleep apnoea patients http://ow.ly/UNchD

The widespread recognition that obstructive sleep apnoea (OSA) represents an important risk factor for motor vehicle accidents (MVA), which is reversed by successful therapy with continuous positive airway pressure (CPAP), has led to a revision of annex III of the European Union (EU) directive on driving licences that is subject to mandatory implementation by all member states from December 31, 2015 [1]. This directive was the result of recommendations from a working group established by the Transport and Mobility Directorate of the European Commission in 2012 [2]. The directive states [1]:

- Applicants or drivers in whom a moderate or severe obstructive sleep apnoea syndrome is suspected shall be referred for further authorised medical advice before a driving licence is issued or renewed. They may be advised not to drive until confirmation of the diagnosis.

- Driving licences may be issued to applicants or drivers with moderate or severe obstructive sleep apnoea syndrome who show adequate control of their condition and compliance with appropriate treatment and improvement of sleepiness, if any, confirmed by authorised medical opinion.

- Applicants or drivers with moderate or severe obstructive sleep apnoea syndrome under treatment shall be subject to a periodic medical review, at intervals not exceeding three years for drivers of group 1 [noncommercial drivers] and one year for drivers of group 2 [commercial drivers], with a view to establish the level of compliance with the treatment, the need for continuing the treatment and continued good vigilance.

While the recognition of OSA and its potential consequences on driving represents a major step towards increased safety on the road, European pulmonologists and sleep specialists face several problems. First, practical application of the directive is demanded from governments of member states. Since rules for medical assessment before obtaining a driving licence differ among European states [3], a standardised approach to implementation of the EU directive would be highly desirable. Second, patients with diagnosed OSA represent the tip of the iceberg of a large population with unrecognised and untreated sleep disordered breathing, and the new requirements established by the EU directive could considerably

This article is published in both the European Respiratory Journal and the Journal of Sleep Research. The articles are identical except for minor stylistic and spelling differences in keeping with each journal's style.

Received: Nov 132015 | Accepted: Nov 162015

Conflict of interest: None declared.

Copyright $\odot$ ERS and @European Sleep Research Society 2016 
increase the number of requests for specialist evaluation, and lengthen waiting lists. Affordable medical evaluations, and an acceptable timeframe to obtain the new or renewed driving licence, appear reasonable targets, but screening for OSA on a large scale will put considerable strain on health systems throughout Europe. Third, episodes of sleepiness at the wheel in the previous 2 years were reported by $17 \%$ of European drivers in a recent survey, underlining that it is quite a common problem [4]. Sleepiness at the wheel was associated with poor sleep, younger age, male sex, driving exposure, daytime sleepiness and a high risk of OSA [4].

Although OSA increases the risk of traffic accidents [5], the disorder is associated with excessive daytime sleepiness (EDS) in only $\sim 50 \%$ of patients. While reports differ, the majority of evidence supports the view that driving risk in OSA is more closely related to the degree of daytime sleepiness than the objective severity of sleep disordered breathing as measured by the frequency of apnoeas and hypopnoeas per hour of sleep (apnoea-hypopnoea index) [6]. However, other factors can contribute to sleepiness in patients with OSA, which include inadequate sleep time, time of day (early morning and afternoon), shift work, sedative medications, poor sleep hygiene, other sleep disorders, and alcohol intake [7]. These additional factors may be particularly important in commercial drivers. Furthermore, these factors, which are not directly related to OSA severity, may contribute to some of the variability in reported sleepiness in patients with differing levels of disease severity based on apnoea-hypopnoea index, and of course may also be present in patients without OSA, thus contributing to accident risk. Effective OSA treatment, usually with CPAP, rapidly resolves both apnoea and EDS in the large majority of affected patients $[8,9]$.

A possible way to deal with the problem of driving in OSA patients was proposed by the British Thoracic Society (BTS) in 2014 [10]. The BTS statement on driving licence regulation pragmatically focused on the evaluation and treatment of sleepy patients only [10]. However, UK subjects requesting a driving licence receive detailed information about OSA symptoms, and have to report symptoms of sleepiness to their general practitioner (GP). Moreover, the Driver and Vehicle Licensing Agency directly manages the assessment, together with the GP or a specialist, if necessary [10]. Such a model is hardly applicable in most EU member states, where the GP or specialist has to issue a medical certificate.

Subjective EDS in OSA patients is usually assessed by questionnaires, which are subjective and thus susceptible to reporting bias by a driver who seeks to underestimate severity, whereas objective evaluation is expensive, time consuming, and not well suited to being performed on a large scale. It is likely that the EU directive will foster research to develop new tools to assess fitness to drive. Such tools are currently lacking, and this is a major problem to face in the near future.

This lack of tools is particularly evident in the context of the assessment of sleepiness. The need for practical guidelines for clinicians is particularly evident in a recent report demonstrating a lack of consensus in clinicians' judgement of fitness to drive in both untreated and CPAPtreated patients with OSA [11].

Because of the major impact the directive might have on sleep and pulmonary specialists, the European Respiratory Society (ERS) and the European Sleep Research Society (ESRS) will jointly appoint a group of experts to develop practical recommendations to cope with the problems raised by the new EU directive on issuing driving licences in Europe. The European Lung Foundation (ELF) will also be involved, providing the important point of view of patients with OSA. Patient involvement is particularly important in order to minimise the risk of encouraging OSA patients to avoid seeking medical attention and treatment because of the understandable concern that such a diagnosis would compromise their ability to continue driving. This risk is particularly concerning for commercial drivers who depend on retention of a valid driving licence for their livelihood and also represent the group where failure to diagnose and treat OSA carries the greatest risk to public safety. In this context, a "carrot and stick" approach is required with the principal emphasis being on the carrot, and a detailed campaign to educate patients, employers and other relevant stakeholders about this topic is required.

Questions to be addressed by the group include the following. How should the suspicion of moderate or severe OSA be justified? Are there simple and readily available tools to assess OSA and sleepiness in the population? What is the consensus on a minimal medical expert standard to establish good compliance to, and effectiveness of, treatment? Which tests or investigations should be used to certify continued good vigilance? In addition to this document, planned for early 2016, further initiatives will be set up to obtain additional information over time, and update/adjust recommendations.

\section{References}

1 European Union. Commission Directive 2014/85/EU of 1 July 2014, amending Directive 2006/126/EC of the European Parliament and of the Council on driving licences. OJ L 194, 2.7.2014, pp. 10-13.

2 McNicholas WT, ed. New Standards and Guidelines for Drivers with Obstructive Sleep Apnoea Syndrome: Report of the Obstructive Sleep Apnoea Working Group. Brussels, European Commission, 2013. 
3 Alonderis A, Barbé F, Bonsignore $\mathrm{M}$, et al. Medico-legal implications of sleep apnoea syndrome: driving license regulations in Europe. Sleep Med 2008; 9: 362-375.

4 Gonçalves M, Amici R, Lucas R, et al. Sleepiness at the wheel across Europe: a survey of 19 countries. J Sleep Res 2015; 24: 242-253.

5 Strohl KP, Brown DB, Collop N, et al. An official American Thoracic Society Clinical Practice Guideline: sleep apnea, sleepiness, and driving risk in noncommercial drivers. An update of a 1994 Statement. Am J Respir Crit Care Med 2013; 187: 1259-1266.

6 McNicholas WT, Rodenstein D. Sleep apnoea and driving risk: the need for regulation. Eur Respir Rev 2015; 24: 602-606.

7 Di Milia L, Smolensky MH, Costa G, et al. Demographic factors, fatigue, and driving accidents: An examination of the published literature. Accid Anal Prev 2011; 43: 516-532.

8 Sassani A, Findley LJ, Kryger M, et al. Reducing motor-vehicle collisions, costs, and fatalities by treating obstructive sleep apnea syndrome. Sleep 2004; 27: 453-458.

9 Tregear S, Reston J, Schoelles K, et al. Continuous positive airway pressure reduces risk of motor vehicle crash among drivers with obstructive sleep apnea: systematic review and metaanalysis. Sleep 2010; 33: 1373-1380.

10 British Thoracic Society. Position Statement: Driving and Obstructive Sleep Apnoea (OSA)/Obstructive Sleep Apnoea Syndrome (OSAS). London, British Thoracic Society, 2014.

11 Dwarakanath A, Twiddy M, Ghosh D, et al. Variability in clinicians' opinions regarding fitness to drive in patients with obstructive sleep apnoea syndrome (OSAS). Thorax 2015; 70: 495-497. 